Pacific Journal of Mathematics

POSTNIKOV-DECOMPOSITIONS OF FUNCTORS 


\title{
POSTNIKOV-DECOMPOSITIONS OF FUNCTORS
}

\author{
FrIEDRICH-WILHELM BAUER
}

Dedicated to Alexander Dinghas on the occasion of his 60 th birthday

The purpose of a Postnikov-decomposition of a covariant functor $\Phi: \Re \rightarrow \mathfrak{C}$ is to approximate $\Phi$ by simpler functors $\Psi: \Re \rightarrow(\sqrt{ }$ (so called Postnikov-functors for $\Phi$ ) which reflect certain properties of $\Phi$ but are simpler to handle. There is always a functor transformation $T: \Phi \rightarrow \Psi$ and a "coincidencecategory" $\mathfrak{Z}_{F}=\{X \mid X \in \Re, T: \mathscr{\Phi}(X) \approx \Psi(X)\}$. A Postnikov-resolution $\mathfrak{U}$ is a family of Postnikov-functors for $\Phi$. There is always a maximal Postnikov-resolution. Under certain conditions each object $X \in \Re$ can be decomposed into a family of objects $\left\{X_{\psi}\right\}$ where $X_{\Psi} \in \mathfrak{S}_{\Psi}$ and $\Psi \in \mathfrak{U}$. The whole theory can be dualized and one gets the theory of dual Postnikov-resolutions. Let $\pi=\Phi$ be the homotopy-functor, $\pi=\left\{\pi_{m}\right\}$; it turns out, that the maximal Postnikov-decomposition of $\pi$ gives exactly the classical theory of Postnikov-complexes of topological spaces or Kan-complexes. A similar result is proved for $\emptyset=$ $H=\left\{H_{m}\right\}$, the homology functor and dual Postnikov-resolutions as well as for dual Postnikov-resolutions of $\pi$ and their connection to Cartan-Serre-fibrations.

The original idea of M. M. Postnikov [7], to build up a space $X$ by simpler spaces $X^{(n)}$ in order to characterize its homotopy type, has been extended, generalized and clarified in many different directions. First of all there is J.C. Moore's natural approach in the category of Kan complexes [6]. D. W. Kahn gave, in a series of more recent papers, answers to many geometrical problems concerning the naturality of Postnikov decompositions (see e.g. [5]). A. Dold [4] succeeded in decomposing a given half exact functor in a way similar to Postnikov's. There appear in the literature many different types of Postnikov-theories which are to a certain extend dual to Postnikov's idea. There is, for instance, the theory of Cartan-Serre-fibrations which was worked out for css-spectra by D. Burghelea and A. Deleanu [3] and which gives a satisfactory dualization of a Postnikov decomposition of a css-spectrum. There is also the (nonnatural) co-Postnikovtheory of B. Eckmann and P. Hilton, where homotopy is replaced by homology. In the author's opinion all these different theories need a common categorical approach to clear up what a Postnikov-decomposition of "something" in fact is.

We will start with an arbitrary convariant functor $\Phi: \AA \rightarrow \sqrt{ }$ where $\mathfrak{K}$ and $\sqrt{S}$ are arbitrary categories. We investigate certain functors $\Psi: \mathscr{K} \rightarrow \mathfrak{C}$ which are closely related to $\Phi$ and which are called "Postnikov- 
functors" for $\Phi$. (Definition 1.2); a family $\mathfrak{A}$ of Postnikov functors is called a resolution of the given functor. We then define a Postnikovsystem (in a given resolution) and a Postnikov decomposition of a given object. The main theorems of theoretical interest are theorems 1 and 2 where a given object is characterized by its Postnikov-system.

All of this can easily be dualized in a categorical way ( $\$ 3)$. In the next four sections we prove that all known Postnikov-theories can be subsumed under our general scheme: The known Postnikovdecompositions of the functor in question are Postnikov-decompositions in our sense and there are no others. The functors which are discussed in this connection are: The homotopy functor $\pi$ with its classical Postnikov decomposition and with its dual (which is closely related to the theory of Cartan-Serre-fibrations); the homology functor and a dual Postnikov-theory. In the last section ( $\$ 9)$ we treat the theory of filtered spaces in connection with a suitable functor $\Lambda$.

We are not going to give new algebraic invariants or to improve the known investigations on Postnikov's original theory. The only purpose of this paper is to give a general frame work in which everything that deserves the name "Postnikov-theory" seems to fit.

1. Postnikov-functors. Let $\Phi: \Re \rightarrow \Subset$ be a given covariant functor. We are going to define what we call a decomposition functor $\Psi: \Re \rightarrow \sqrt{ }$ of $\Phi$.

Definition 1.1. A triple $\alpha=(\Psi, T, P)$ where $T: \Phi \rightarrow \Psi$ and $P: \Psi \rightarrow$ $\Phi$ are transformations is called a $d$-functor for $\Phi$ if the following conditions are fulfilled:

(d1) $T P=1:$

(d2) If $\Phi(f)$ is an isomorphism for $f: X \rightarrow Y$ in $\Re$, then $\Psi(f)$ is an isomorphism.

We consider the full subcategory $\&_{\alpha} \subset \Omega$ defined by the following objects:

$$
\mathfrak{Q}_{\alpha}=\{X \mid X \in \Re, T: \Phi(X) \approx \Psi(X)\} .
$$

Definition 1.2. A $d$-functor $\alpha=(\Psi, T, P)$ for $\Phi$ is a Postnikovfunctor for $\Phi$ if $\alpha$ satisfies the following two conditions:

(P1) For each $X \in \Re$ there is a map $k: X \rightarrow X_{\alpha}$ such that

$$
X_{\alpha} \in \mathfrak{R}_{\alpha} \text { and } \Psi(k)=\text { isomorphism . }
$$

(P2) If $k^{\prime}: X \rightarrow X_{\alpha}^{\prime}$ is another map which fulfills (P1), then there is a unique map $r: X_{\alpha} \rightarrow X_{\alpha}^{\prime}$ with $r k=k^{\prime}$.

Obviously $\Phi(r)$ is an isomorphism.

A $d$-functor which fulfills only (P1) is called a "weak-Postnikovfunctor". 
Let $\alpha=(\Psi, T, P), \beta=\left(\Omega, T_{1}, P_{1}\right)$ be two $d$-functors to $\Phi$. We write $\alpha \leqq \beta$ if there is a factorization of functor-transformations:

$$
T=S T_{1}, \quad P=P_{1} Q
$$

such that $1=S Q$, and if $\Omega(f)=$ isomorphism, $f \in k$ implies $\Psi(f)=$ isomorphism.

1.1. One has $\alpha \leqq \alpha ; \alpha \leqq \beta, \beta \leqq \gamma \Rightarrow \alpha \leqq \gamma$; if $\alpha \leqq \beta \leqq \alpha$ then $S$ is a functor isomorphism and $Q$ is its inverse.

Proof. Only the last assertion needs proof. In addition to $Q$ and $S$ there are transformations $\bar{Q}: \Omega \rightarrow \Psi$ and $\bar{S}: \Psi \rightarrow \Omega$ such that

$$
P \bar{Q}=P_{1}, \bar{S} T=T_{1}, \bar{S} \bar{Q}=1 .
$$

Thus the following identities hold:

$$
S=T P_{1}=Q, Q=T_{1} P=S .
$$

Therefore $Q S=1$ and this proves 1.1..

1.2. If $\alpha \leqq \beta$ then $\mathfrak{Q}_{\alpha} \subseteq \mathfrak{Q}_{\beta}$.

Proof. On $\mathfrak{R}_{\alpha}$ the transformation $T$ is an isomorphism and $P$ is its inverse. Since $T=S T_{1}$, we have established the equality $(P S) T_{1}=$ 1. But $S=T P_{1}$ (everything on $\mathbb{2}_{\alpha}$ ) and thus $T_{1}(P S)=T_{1} P T P_{1}=T_{1} P_{1}=1$. Therefore $T_{1}$ is an isomorphism on $\mathfrak{Q}_{\alpha}$, proving the desired inclusion.

The greatest Postnikov-functor is obviously $\mu=(\Phi, 1,1), 1: \Phi \rightarrow \Phi$ where $\mathfrak{L}_{\mu}=\Re$ holds.

Definition 1.3. By a Postnikov resolution of functor $\Phi$ we mean a family $\mathfrak{U}=\{\alpha\}$ of Postnikov-functors for $\Phi$.

This is the essential definition which will enable us to define Postnikov-systems and Postnikov-decompositions of an object $X \in \Re$. It is not necessarily required that $\mathfrak{A}$ contain all Postnikov-functors for $\Phi$.

Let $\Phi: \Re \rightarrow \mathfrak{S}, A: \mathbb{C} \rightarrow \mathbb{C}^{\prime}$ be convariant functors. If $\Psi: \Re \rightarrow \mathfrak{S}$ is a $d$-functor for $\Phi$, one sees immediately that $\Psi^{\prime}=\Lambda \Psi$ fulfills (d1) for $\Phi^{\prime}=\Lambda \Phi$. The same is true for weak Postnikov-functors: If $\Psi$ is a weak Postnikov-functor for $\Phi$ we conclude that $\Psi^{\prime}$ is a weak Postnikov-functor to $\Phi^{\prime}$ provided (d2) holds. A corresponding statement for (P2) is only true under the following restriction on $\Phi$ :

(*) If $\varphi \in \mathfrak{C}, \Lambda(\varphi)$ is an isomorphism, then $\varphi$ itself is an isomorphism. In case $(*)$ is valid, $\mathfrak{R}_{\alpha}$ will be transformed onto $\mathfrak{R}_{\alpha}^{\prime}=\{X \mid X \in \Re$, $\Lambda(T): \Lambda \Phi(X) \approx \Lambda \Psi(X)\}$, which proves (P2) for $\Psi^{\prime}$ and $\Phi^{\prime}$. We have therefore proved: 
Let $\Phi: \Re \rightarrow \sqrt{ }$ and $\Lambda: \mathbb{C} \rightarrow \sqrt{ }{ }^{\prime}$ be covariant functors such that $(*)$ is true if $\alpha=(\Psi, T, P)$ is a (weak) Postnikov-functor for $\Phi$, then $\alpha^{\prime}=(\Lambda \Psi, \Lambda T, \Lambda P)$ is a (weak) Postnikov functor for $\Lambda \Phi$. Moreover, whenever $(*)$ is fulfilled, $\alpha^{\prime}$, is in fact a Postnikov functor for $\Lambda \Phi$.

2. Postnikov-systems and Postnikov-decompositions. Let $\mathfrak{A}=$ $\{\alpha\}$ be a Postnikov-resolution of $\Phi$ and let $\alpha \leqq \beta$ in $\mathfrak{A}$. We consider mappings $h=h_{\alpha}^{\beta}: X_{\beta} \rightarrow X_{\alpha}$ such that

$$
X_{\alpha} \in \mathfrak{Q}_{\alpha}, X_{\beta} \in \mathfrak{R}_{\beta}
$$

$\Psi_{\alpha}(h)$ is an isomorphism, $\alpha=(\Psi, T, P)$.

We will need the following condition:

(I) If $\Phi(f)$ is an isomorphism in $\sqrt{5}$ then $f$ is an isomorphism in $\Omega$.

In the fourth section we will discuss the nature of this assumption.

Definition 2.1. A Postnikov-system $\mathfrak{a}=\left\{h_{\alpha}^{\beta}\right\}$ consists of an object $X_{\alpha} \in \mathfrak{R}_{\alpha}$ for each $\alpha \in \mathfrak{A}$ and a map $h=h_{\alpha}^{\beta}: X_{\beta} \rightarrow X_{\alpha}$ for each $\alpha \leqq \beta$, such that

(1) $h_{\alpha}^{\alpha}=$ identity

(2) $\alpha \leqq \beta \leqq \gamma \Rightarrow h_{\alpha}^{\beta} h_{\beta}^{\gamma}=h_{\alpha}^{\gamma}$,

i.e., a Postnikov-system is a projective spectrum with connecting maps $h_{\alpha}^{\beta}$.

Let $\mathfrak{A}$ be a given resolution of $\Phi$; we define $\overline{\mathfrak{U}}=\mathfrak{A} \cup \mu$ to be the resolution obtained from $\mathfrak{U}$ by just adding $\mu$ as terminal object. A Postnikov decomposition of a given object $X \in \Re$ in $\Re$ is a Postnikovsystem $\overline{\mathfrak{a}}_{X}=\mathfrak{a}=\left\{h_{\alpha}^{\beta}\right\}$ in $\overline{\mathfrak{U}}$ such that $X_{\mu}=X$. We will prove the existence of $\overline{\mathfrak{a}}_{X}$ under condition (I): By Definition 1.2. there is for each $\alpha \in \mathfrak{U}$ a map $h_{\alpha}=h_{\alpha}^{\mu}: X \rightarrow X_{\alpha}$ with the properties stated in (P1). If $\alpha \leqq \beta$ in $\mathfrak{A}$, we have a diagram:

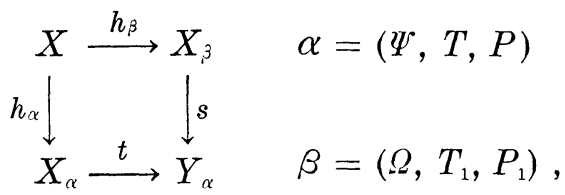

where $\Psi(s)$ is an isomorphism, $Y_{\alpha} \in \mathfrak{R}_{\alpha}$, and $t$ is in $\mathfrak{Z}_{\alpha}$. We define $h_{\alpha}^{\beta}=t s$, which is obviously a map with $\Psi\left(h_{\alpha}^{\beta}\right)=$ isomorphism. The map $h_{\alpha}^{\beta}$ apparently depends on the choice of $s$ and $t$; we prove the independence of $t s$. Let $\bar{s}: X_{\beta} \rightarrow \bar{Y}_{\alpha}$ and $\bar{t}: \bar{Y}_{\alpha} \rightarrow X_{\alpha}$ be a different pair of maps. We then have a diagram of the following kind: 


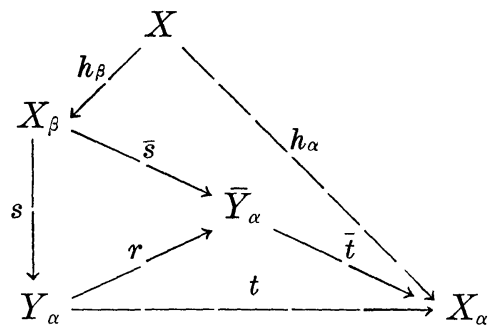

where $r$ comes from (P2) in Definition 1.2. and makes this diagram commutative. This implies that $t s=\bar{t} \bar{s}$. Now let $\alpha \leqq \beta \leqq \gamma$ in $\mathfrak{A}$; the proof that $h_{\alpha}^{\beta} h_{\beta}^{\gamma}=h_{\alpha}^{\gamma}$ runs along the same line.

This completes the existence proof of a Postnikov-system $\overline{\mathfrak{a}}_{X}$ for given $X \in \Re$.

2.1. If condition ( $\mathrm{I}$ ) is fulfilled, then there exists a Postnikovdecomposition $\overline{\mathfrak{a}}_{X}$ in $\mathfrak{A}$ for each $X \in \Re$. There may exist of course, more than one $\overline{\mathfrak{a}}_{X}$ for a given $X \in \Re$; we must therefore compare two different Postnikov-decompositions of the same object $X \in \Re$. For this purpose we introduce the notion of an isomorphism $k=\left\{k_{\alpha}\right\}$ for two Postnikov-systems $\mathfrak{a}$ and $\tilde{\mathfrak{a}}$. If $\mathfrak{a}=\left\{h_{\alpha}^{\beta}\right\}, \tilde{\mathfrak{a}}=\left\{\tilde{h}_{\alpha}^{\beta}\right\}$, an isomorphism $k_{\beta}=$ $\left\{k_{\alpha}\right\}: \mathfrak{a} \rightarrow \tilde{\mathfrak{a}}$ is a family of maps $k_{\alpha}: X_{\alpha} \rightarrow \bar{X}_{\alpha}$ such that $k_{\alpha} h_{\alpha}^{\beta}=\widetilde{h}_{\alpha}^{\beta} k_{\beta}$ and

$$
\Psi\left(k_{\alpha}\right)=\text { isomorphism . }
$$

2.2. Let $\tilde{\mathfrak{a}}_{X}, \mathfrak{a}_{X}$ be two Postnikov-systems for a fixed $X \in \Re$. Then there exists a unique isomorphism $k=\left\{k_{\alpha}\right\}: \mathfrak{a}_{X} \rightarrow \tilde{\mathfrak{a}}_{X}$ such that $k_{\mu}: X \rightarrow X$ is the identity.

Proof. Consider the diagram

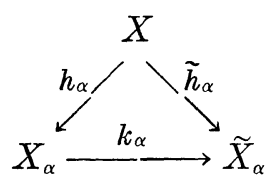

where $k_{\alpha}$ exists by Definition 1.2. Obviously $\Psi\left(k_{\alpha}\right)$ is an isomorphism and $k_{\alpha}$ unique. One has to prove that the diagram:

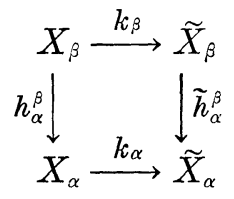

is commutative. Again by Definition 1.2. we get a map $m_{\alpha}: X_{\alpha} \rightarrow \widetilde{X}_{\alpha}$ such that 


$$
m_{\alpha} h_{\alpha}^{\beta}=\widetilde{h}_{\alpha}^{\beta} k_{\beta} .
$$

The commutativity of

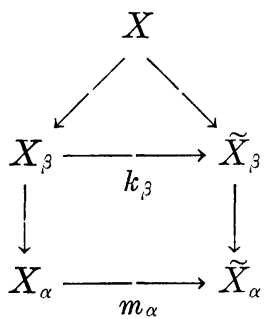

and the uniqueness of $k_{\alpha}$ implies that $k_{\alpha}=m_{\alpha}$. Thus $k=\left\{k_{\alpha}\right\}$ is an isomorphism of $\mathfrak{a}_{X}$ and $\tilde{\mathfrak{a}}_{X}$ with the desired properties.

2.3. Let $f: X \rightarrow \widetilde{X}$ be a map in $\Re$ with $\Phi(f)=$ isomorphism an $a_{X}$ a Postnikov-decomposition of $X, a_{\tilde{X}}$ a Postnikov-decomposition of $\tilde{X}$. There is a unique isomorphism $k=\left\{k_{\alpha}\right\}: a_{X} \rightarrow a_{\tilde{Y}}$ such that $k_{n !}: X \rightarrow$ $\tilde{X}$ is exactly $f$.

The proof is a mere repetition of the arguments used in the proof of 2.2.

TheOREm 1. Let $\mathfrak{A}$ be a resolution of $\Phi$, and let $\overline{\mathfrak{A}}=\mathfrak{A} \cup \mu$. If (I) is true, then there exists for each $X \in \Re$ a Postnikov-decomposition $\overline{\mathfrak{a}}_{X}$. If $f: X \rightarrow \tilde{X}$ in $\Re$ and $\Phi(f)$ is an isomorphism one can find an isomorphism $k=\left\{k_{\alpha}\right\}: \overline{\mathfrak{a}}_{X} \rightarrow \overline{\mathfrak{a}}_{\tilde{X}}$ of Postnikov-systems, which is uniquely determined by $k_{\mu}=f$.

Let $\overline{\mathfrak{a}}_{X}$ be a Postnikov-decomposition of an object $X$ in $\Re$. By dropping all those terms in $\overline{\mathfrak{a}}_{X}$ which involve $\mu=(\Phi, 1,1)\left(\right.$ e.g. $X_{\mu}=X$, $h_{\alpha}: X_{\mu} \rightarrow X_{\beta}$ etc.) we get a Postnikov-system in $\mathfrak{A}$ which we call $\mathfrak{a}_{X}$. In case $\mu$ is already contained in $\mathfrak{A}$, and therefore $\overline{\mathfrak{A}}=\mathfrak{A}$, we define $\mathfrak{a}_{X}=\overline{\mathfrak{a}}_{X}$.

A Postnikov-system $\mathfrak{a}$ in $\mathfrak{A}$ is called convergent (or convergent to $X)$ if there is an object $X \in \Re$ with $\mathfrak{a}_{X}=a$.

To give a first application of our theory we assume $\sqrt{5}$ to be the category of sets:

Definition 2.2. A resolution $\mathfrak{A}$ is called complete if the following is true:

(1) $\Phi(X)=\bigcup_{\alpha} P \Psi(X), X \in \Re$ for all $\alpha=(\Psi, T, P) \in \mathfrak{N}$.

(2) Let $X, X^{\prime} \in \Re$, let $\mathfrak{a}_{X}, \mathfrak{a}_{X^{\prime}}$ be two Postnikov-decompositions of $X$ resp. $X^{\prime}$ and $k=\left\{k_{\alpha}\right\}: a_{X} \rightarrow a_{X}$ an isomorphism. Then there is a map 
$f: X \rightarrow X^{\prime}$ in $\mathfrak{K}$ such that all diagrams

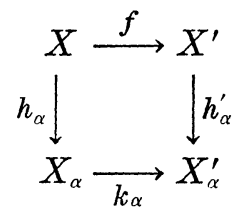

are commutative.

(3) If $\alpha, \beta \in \mathfrak{A}$ then there is $\gamma \in \mathfrak{A}$ such that $\gamma \geqq \alpha, \beta$.

One can easily prove:

2.4. Let $\mathfrak{A}$ be a complete resolution, and let $a, a^{\prime}$ be convergent and isomorphic (i.e. $a=a_{x}, a^{\prime}=a_{x^{\prime}}, k: a_{x} \rightarrow x^{\prime}$ an isomorphism). Then there is an isomorphism $\bar{k}: \overline{\mathfrak{a}}_{X} \rightarrow \overline{\mathfrak{a}}_{X^{\prime}}$ which extends $k$.

Proof. Note that $k_{\mu}: X_{\mu}=X \rightarrow X_{\mu}^{\prime}=X^{\prime}$ is the map $f$ and that $\Phi(f)$ an isomorphism by Definition 2.2. (1), (3).

Theorem 2. Given two objects $X, X^{\prime} \in \mathfrak{K}$ and Postnikov decompositions $\mathfrak{a}_{X}, \mathfrak{a}_{X^{\prime}}$ in a complete resolution $\mathfrak{A}$. The following two conditions are equivalent:

(1) $a_{X}$ and $a_{X}$, are isomorphic,

(2) there is a map $f: X \rightarrow X^{\prime}$ such that $\Phi(f)$ is an isomorphism. This is the root of a classical theorem on the determination of the homotopy type of a space by its Postnikov decomposition.

3. Dualization. Everything that was developed in $\S 1$ and $\S 2$ can be dualized in several different ways:

First, one can replace covariant functors by contravariant functors. This type of dualization is not so interesting from the theoretical point of view.

Second, one can dualize formally: $A$ (dual) $d$-functor to $\Phi$ is a triple $(\Psi, T, P)$ where $T: \Psi \rightarrow \Phi, P: \Phi \rightarrow \Psi$ with $P T=1$ and (d2) in Definition 1.1. In spite of the fact that every dual $d$-functor is itself a $d$-functor (by exchanging the roles of $P$ and $T$ ) and vice-versa, we will preserve our notations (of $P$ and $T$ ) for dual $d$-functors.

The dualization of a Postnikov-functor is something more substantial. (P1) is replaced by:

( $\widetilde{\mathrm{P}} 1)$ For given $X \in \Re$ there is a map $k: X_{\alpha} \rightarrow X$ such that $X_{\alpha} \in \mathbb{R}_{\alpha}$ and $\Psi(k)$ is an isomorphism.

and (P2) by:

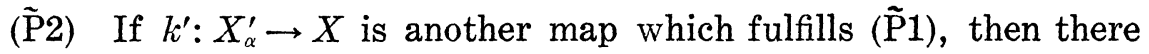


is an unique $r: X_{\alpha}^{\prime} \rightarrow X_{\alpha}$ with $k r=k^{\prime}$.

Because this dualization process is categorical, all theorems have a dual counterpart which does not need an extra proof.

4. The category $\mathfrak{R}^{\Phi}$. Let $\Re$ be a given category and $\Phi: \Re \rightarrow \mathfrak{C}$ a covariant functor. We defined in [1] a category $\Re^{\oplus}$ and a functor $\eta: \Omega \rightarrow \Omega^{\Phi}$ with the following properties:

( $\eta 1) \quad \eta$ is the identity on the objects.

$(\eta 2)$ If $\phi(f)$ is an isomorphism, then $\eta(f)$ is an equivalence.

( $\eta 3)$ If $\lambda: \Omega \rightarrow \Omega$ is any functor with the properties of $\eta$ (i.e. $\eta 1)$, $\eta 2)$ ) there is a unique functor $\rho: \Omega^{\Phi} \rightarrow \Omega$ with $\rho \eta=\lambda$.

Since $\Phi$ itself has properties $(\eta 1),(\eta 2)$ (after a suitable interpretation of $\Phi$ on the objects: two different objects in $\Omega$ are always mapped into different (but maybe equivalent) objects in (5) there is a uniquely determined functor $\Phi^{\prime}: \Re^{\Phi} \rightarrow \mathbb{C}$ with $\Phi^{\prime} \eta=\Phi$. Later on we will have to deal with functors such that $\eta$ is epimorphic in the following sense:

If $\alpha: X \rightarrow Y$ is a map in $\AA^{\Phi}$ there is a $f: X \rightarrow Y$ in $\Re$ with $\eta(f)=\alpha$. Under this restriction, the functor $\Phi^{\prime}: \mathscr{\Re}^{\Phi} \rightarrow \mathfrak{C}$ has property (I) in last section:

$\Phi^{\prime}(f)$ an isomorphism if and only if $f$ is an equivalence in $\Re^{\Phi}$. In the next section we will meet categories with homotopy where $\eta$ turns out to be an epimorphism of this type.

5. The functor $\pi$. There are numerous categories in topology and algebra where a homotopy functor is defined. All these functors have in common the image category $\mathbb{C}=\mathbb{B}$ shere $\mathbb{B}$ is the category of group systems $G=\left\{G_{n}\right\}, n=0,1, \cdots$ where $G_{n}$ is a group and $\phi=\left\{\varphi_{n}\right\}:\left\{G_{n}\right\} \rightarrow\left\{F_{n}\right\}$ is a family of homomorphisms $\varphi_{n}: G_{n} \rightarrow F_{n}$. We will be concerned with the following categories:

(1) $\Omega=\wp_{E}=$ category of css-complexes with Kan condition (i.e. Kan's extension condition [6]).

(2) $\Omega=\mathfrak{I}$ category of all topological spaces and continuous maps.

(3) $\Re=\mathfrak{I}_{0}$ based spaces and basepoint preserving maps.

(4) $\Omega=\mathfrak{I}_{s}$ simply connected spaces

( 5 ) $\quad \Re=\mathfrak{P}_{0}, \mathfrak{P}_{s, \ldots}$. The same as in (2)-(4) but $C W$-spaces instead of arbitrary spaces.

6. $\Omega=\wp_{p_{\mathrm{E}}}$ css-spectra with extension condition [3]. All these categories are equipped with a homotopy. Thus it makes sense to speak of the homotopy type of a given object. The homotopy functor which is defined (in different ways) for all of these categories has for $\wp_{\mathrm{E}}, \wp_{p_{\mathrm{E}}}, \Re_{0}$ the following property (Whitehead's theorem):

(W) $A \operatorname{map} f: X \rightarrow Y$ is $\Re$ is a homotopy equivalence if and only if $\pi(f)$ is an isomorphism. 
We will prove that under this condition the category $\Omega^{\pi}$ is isomorphic to the category $\Re_{H}$, the homotopy cotegory of $\Re$ (objects are as in $\Re$ but maps are homotopy classes of maps in $\Re$ ). In other words: Two maps $f_{0}, f_{1}: X \rightarrow Y$ in $\Re$ are homotopic, $f_{0} \cong f_{1}$, if and only if $\eta\left(f_{0}\right)=\eta\left(f_{1}\right)$. The proof is rather easy: Consider the natural functor $H: \Omega \rightarrow \Re_{H}$ and verify conditions $(\eta 1),(\eta 2)$ in the preceding paragraph. There is a unique functor $\rho: \AA^{\pi} \rightarrow \Re_{H}$ with $\rho \eta=H$. Now take two maps $f_{0}, f_{1}: X \rightarrow Y$ which are homotopic. In all cases (1)-(6) there is an object $P_{X}$ in $\Re$, maps $i_{0}, i_{1}: X \rightarrow P_{X}, r: P_{X} \rightarrow X, F: P_{X} \rightarrow Y$ such that:

$$
\begin{aligned}
& i_{j}, r \text { are homotopy equivalences } \\
& r i_{0}=r i_{1} \\
& f_{j}=F i_{j}, j=0,1 .
\end{aligned}
$$

Since $r$ is a homotopy equivalence it follows $\eta\left(i_{0}\right)=\eta\left(i_{1}\right)$ and therefore

$$
\eta\left(f_{0}\right)=\eta(F) \eta\left(i_{0}\right)=\eta(F) \eta\left(i_{1}\right)=\eta\left(f_{1}\right) \text {. }
$$

This shows that there is a functor $\bar{\rho}: \mathfrak{R}_{H} \rightarrow \Re^{\pi}$ which fulfills $(\eta 1)$ and $\bar{\rho} H=\eta$ and proves:

5.1. In a category $\Re$ where $\pi$ fulfills $W$ ), for two maps $f_{0}, f_{1}: X \rightarrow Y$ in $\Re$ the following two conditions are equivalent:

(a) $\eta\left(f_{0}\right)=\eta\left(f_{1}\right)$

(b) $f_{0} \cong f_{1}$.

The candidates for a Postnikov-decomposition of $\pi$ are the functors

$$
\pi^{(n)}=\left\{\pi_{m}\right\}_{m \leqq n}
$$

in other words: $\pi^{(n)}=\left\{G_{m}\right\}$, where $G_{m}=1$ for $m>n$ (homotopy groups are written multiplicatively) and $G_{m}=\pi_{m}$ for $m \leqq n$. One proves easily:

5.2. $\alpha=\left(\pi^{(n)}, T^{(n)}, P^{(n)}\right)$ is a $d$-functor for $\pi$ where $T^{(n)}$ resp. $P^{(n)}$ are the projection resp. the inclusion.

The verification of (P1), (P2) is done for the category $\wp_{\mathrm{E}}$. By applying the functors $R: \wp_{\mathrm{E}_{0}} \rightarrow \mathfrak{P}_{0}$ and $S: \mathfrak{P}_{0} \rightarrow \wp_{\mathrm{E}_{0}}$ (realization and singular complex) one easily obtains properties (P1), (P2) for $\mathfrak{\beta}_{0}$ itself. To each $X \in \wp_{E_{0}}$ we find an $n$-stage Postnikov-decomposition [6] in the classical sense, i.e., a fibre map $p: X \rightarrow X^{(n)}$ where $\pi_{m}(p)$ is an isomorphism for $m \leqq n$ and $\pi_{m}\left(X^{(n)}\right)=1$ for $m>n$. Since $X^{(n)}$ is contained in $\mathfrak{R}_{\alpha}=$ $\left\{X \mid \pi_{m}(X)=1, m>n\right\}$ we obtain (P1). Now let $X_{1}$ be any object in $\mathfrak{R}_{\alpha}$ and $k_{1}: X \rightarrow X_{1}$ a suitable map which fulfills (P1). By the naturality of Postnikov-decompositions (in the classical sense) we get in $\gamma_{E_{0}}$ a commutative diagram: 


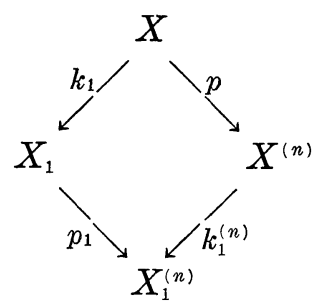

where $p_{1}$ is the $n$-stage Postnikov-map for $X_{1}$ and $k_{1}^{(n)}$ is induced by $k_{1}$. However $p_{1}$ is a homotopy equivalence (since $X_{1} \in \mathfrak{R}_{\alpha}$ ) and we therefore have a map $r: X^{(n)} \rightarrow X_{1}$ such that $r p \cong k_{1}$. If $\bar{r}$ is another map in $\wp_{\mathrm{E}_{0}}$ with $\bar{r} p \cong k_{1}$ we have to show that $r \cong \bar{r}$. On $X_{n}$, the $n$-skeleton of $X$ the map $p$ is an isomorphism (by the construction of $p$ by J.C. Moore [6]). Therefore $\bar{r}\left|\left(X^{(n)}\right)_{n}=r\right|\left(X^{(n)}\right)_{n}$. On the higher dimensional skeleton $r$ and $\bar{r}$ are again homotopic since there are no nontrivial homotopy groups and consequently no obstructions against the extension of a homotopy.

This completes the proof of:

5.3. In the categories $\wp_{\mathrm{EOH}}$ and $\mathfrak{P}_{\mathrm{OH}}, \alpha=\left(\pi^{(n)}, T^{(n)}, P^{(n)}\right)$ is a Postnikov-functor for $\pi$.

5.4. The statement of 5.3 remains true if we replace $\gamma$ by $\gamma_{p_{E}}$. See [3] where a Postnikov-theory for $\gamma_{p_{E}}$ is developed. Details are left to the reader. See [3] for further references.

6. All Postnikov-decompositions of $\pi$. In the last section we proved that all functors $\pi^{(n)}$ are Postnikov-functors for $\pi$ (with $T^{(n)}=$ inclusion). In this section we are going to determine all Postnikovfunctors for $\pi$ (moreover: all weak Postnikov-functors) and it will be proved that they are all of the form $\Psi=\pi^{(n)}$.

Theorem 3. On the category $\mathfrak{I}_{0 s}$ (see last section for definition) every weak Postnikov-functor $\alpha=(\Psi, T, P)$ for $\pi$ is isomorphic either to $\left(\pi^{(n)}, T^{(n)}, P^{(n)}\right)$ or to $(\pi, 1,1)$.

Proof. Let $a \in \pi_{n}(X), T(a) \neq 1$. Then there is a map $f \in a, f: S^{n} \rightarrow X$ with $\pi(f) \nu=a, 1 \in \nu, 1: S^{n} \rightarrow S^{n}$. This implies $T(\nu) \neq 1$. Take $P T: \pi_{n}\left(S^{n}\right) \rightarrow$ $\pi_{n}(S)$. We clearly have $P T(\nu)=\nu^{r}$ where $r \neq 1$. Thus

$$
T(\nu)=T P T(\nu)=T\left(\nu^{r}\right)
$$

and $T\left(\nu^{r-1}\right)=1$.

This implies that $r=1$ since $T$ on $\pi_{n}\left(S^{n}\right)$ has trivial kernel. Thus we have proved $P T(\nu)=\nu$. If $a \in \pi_{n}(X), f \in a$, we get immediately 
the following relation:

$$
P T(a)=\pi(f) P T(\nu)=\pi(f) \nu=a .
$$

In other words: If there is an $a \in \pi_{n}(X), T(a) \neq 1$, then $T$ maps $\pi_{n}(Y)$ for all $Y \in \Re$ injectively into $\Psi(Y)$. Our proof will be finished as soon as we are able to prove that $T\left(\nu^{\prime}\right) \neq 1$ for $\nu^{\prime} \in \pi_{n-1}\left(S^{n-1}\right)$, the homotopy class of the identity (one dimension less). This would mean that the existence of an $a \in \pi_{n}(X)$ with $T(\alpha) \neq 1$ implies that $T$ maps $\pi^{(n)}$ injectively into $\Psi$. We have to discuss two cases:

(1) There is an index $n$ and an $a \in \pi_{n}(X)$ with $T(a) \neq 1$ but no $a^{\prime} \in \pi_{n+1}(\varphi)$ with $T\left(a^{\prime}\right) \neq 1$.

(2) There is no such $n$.

In the first case we get immediately:

$$
\pi^{(n)} \approx \Psi
$$

and in the second case

$$
\pi \approx \Psi
$$

We now prove $T\left(\nu^{\prime}\right) \neq 1$ under our assumptions. Take the Hopf-map $h: S^{n} \rightarrow S^{n-1}(n>2)$ and recall that $b=\pi(h) \nu \neq 1$ and thus $T(b) \neq 1$. Condition (P1) in Definition 1.2. guarantees the existence of an object $X_{1} \in \mathfrak{R}_{\alpha}$ and a map $k: S^{n-1} \rightarrow X_{1}$ such that $\Psi(k)$ is an isomorphism. Since $T(b) \neq 1$ it follows $T(\pi(k) b) \neq 1$ and consequently that $\pi(k) b \neq 1$. Assume $T\left(\nu^{\prime}\right)=1$ ! This implies that

$$
\Psi(k) T\left(\nu^{\prime}\right)=T\left(\pi(k) \nu^{\prime}\right)=1
$$

and therefore $\pi(k) \nu^{\prime}=1$ (since $X_{1} \in \mathfrak{R}_{\alpha}$ ). This is, however, a contradiction because it follows from $\pi(k) \nu^{\prime}=1$ that $k$ is homotopic to the constant map and therefore that $T(\pi(k) b)$ must be trivial. This completes the proof of theorem 3 .

Corollary 1. For the functor $\pi$ all weak Postnikov-functors are Postnikov functors.

REMARK. The proof carries through if we replace $\mathfrak{I}_{0 s}$ by any of the categories $\wp_{E_{0}}$ or $\wp_{p_{E_{0}}}$. Again details are left to the reader.

7. Dual Postnikov-decompositions of $\pi$. The functor $\pi$ on the category $\mathfrak{K}=\mathfrak{T}_{0 s}$ admits a dual Postnikov-decompositions. To show that, we define the functor

$$
\pi_{(n)}=\left\{\pi_{m}\right\}_{m>n}
$$

in other words: 


$$
\pi_{(n)}=\left\{G_{m}\right\}, G_{m}=1 \text { for } m \leqq n, G_{m}=\pi_{m} \text { for } m>n .
$$

For $T_{(n)}: \pi_{(n)} \rightarrow \pi$ and $P_{(n)}: \pi \rightarrow \pi_{(n)}$ we take the inclusion resp. the projection. We get immediately $P_{(n)} T_{(n)}=1$. If $f: X \rightarrow Y$ and $\pi(f)$ is an isomorphism, then so is $\pi_{(n)}(f)$. This proves:

7.1. $\alpha=\left(\pi_{(n)}, T_{(n)}, P_{(n)}\right)$ is a dual $d$-functor to $\pi$.

Now let $\mathfrak{R}_{\alpha}$ be the "coincidence-category": $\mathfrak{L}_{\alpha}=\left\{X \mid \pi_{m}(X)=1\right.$ for $m \leqq n\}$ and let $X \in \Re$ be an arbitrary space. We will construct a map $k: X_{1} \rightarrow X$ with $X_{1} \in \mathfrak{R}_{\alpha}$ and $\pi_{m}(k)=$ isomorphism for $m>n$, in the following way: We know that $R S(X)$, the geometric realization of $S(X)$, admits a Cartan-Serre fibration $X_{1} \stackrel{\rho}{\longrightarrow} R S(X)$ such that $\pi_{m}(p)=$ isomorphism for $m>n$ and $\pi_{m}\left(X_{1}\right)=1$ for $m \leqq n$. Our map $k$ is the composition

$$
k=\omega p
$$

where $\omega$ is the natural weak homotopy equivalence $\omega: R S(X) \rightarrow X$.

7.2. $\alpha=\left(\pi_{(n)}, T_{(n)}, P_{(n)}\right)$ is a weak dual Postnikov functor (i.e., it fulfills $(\tilde{\mathrm{P}} 1))$.

\subsection{Our $\alpha$ is a dual Postnikov-functor to $\pi$.}

Proof. Take any other map $k^{\prime}: X_{2} \rightarrow X$ with $\pi_{m}\left(k^{\prime}\right)=$ isomorphism for $m>n$, and $\pi_{m}\left(X_{2}\right)=1$ for $m \leqq n$. Then there is a map $g_{a}: S_{a}^{m} \rightarrow X_{2}$, $a \in \pi_{m}\left(X_{1}\right)$ such that $k^{\prime} g_{a} \cong k \mid S_{a}^{m}$ which is uniquely determined up to homotopy. Thus the map $g=\bigvee g_{a}: B \rightarrow X_{2}$ can be extended in a unique way to a map $\bar{g}: \bar{B} \cong X_{1} \rightarrow X_{2}$ such that $k \cong k^{\prime} g$.

We are now going to verify the dual statement to Theorem 3 .

Theorem 4. On the category $\mathfrak{I}_{0}$ and $\mathfrak{I}_{0_{I}}$ every weak dual Postnikov-functor $\alpha=(\Psi, T, P)$ for $\pi$ is isomorphic either to $\left(\pi_{(n)}, T_{(n)}, P_{(n)}\right)$ or to $(\pi, 1,1)$.

Proof. The proof is similar to that of Theorem 3. Let $\bar{a} \neq 1$, $\bar{a} \in \Psi(X)$, so we can find an $a=T(\bar{a}) \neq 1$. If $f \in a \in \pi_{n}(X)$ we get $\pi(f) \nu=a$ and $\Psi(f) P(\nu)=a$ and therefore $T P(\nu)=\nu^{r}, r \neq 0$. We will prove the existence of an $\nu \in \Psi\left(S^{n}\right)$ with $T(\bar{\nu})=\nu$. Let $\nu^{r}=T(\bar{b})$, $\bar{b} \in \Psi\left(S^{n}\right)$; we find a map $g: S^{n} \rightarrow S^{n}$ such that $\pi(g) \nu=\nu^{r}$ and such that $\pi(g)$ is injective. Thus, by the same argument which we used before, there is an element $c \in \pi_{n}\left(S^{n}\right)$ which lies in the image of $T$ and has property $\pi(g) c=\nu^{r}$. However under these circumstances we must have $c=\nu$ which proves the existence of a $\nu \in \Psi\left(S^{n}\right)$ with $T(\bar{\nu})=\nu$.

If now $a \in \pi_{n}(Y)$ is an arbitrary element in $\pi_{n}(Y)$ we get imme- 
diately (by the same reasoning) an $\bar{a} \in \Psi(Y)$ with $T(\bar{a})=a$. In other words: If $a \neq 1, a \in \pi_{n}(X)$ is in the image of $T$, the total group $\pi_{n}(Y)$ for arbitrary $Y \in \Re$ is contained in the image of $T$. Now let again $h: S^{n+1} \rightarrow S^{n}$ be the Hopf map ${ }^{1}$ and $k: R \rightarrow S^{n}$ a map, which fulfills all requirements of (P1). Since $\nu \in$ image $\pi_{n}(k)$ there is a map $r: S^{n} \rightarrow R$ with $k r \cong 1: S^{n} \rightarrow S^{n}$. Therefore $r h \not 0$ and there is an $\bar{a} \in \Psi(R)(\approx \pi(R))$ such that $T(\bar{a})=[r h]$. Now we can apply again all the arguments used before and obtain a $\bar{\nu}^{\prime} \in \Psi\left(S^{n+1}\right)$ such that $T\left(\bar{\nu}^{\prime}\right)=\nu^{\prime} \in \pi_{n+1}\left(S^{n+1}\right)$, $1 \in \nu^{\prime}$. The rest of the proof runs as the corresponding part of the proof of Theorem 3 .

Corollary 1. For the functor $\pi$ all dual weak Postnikov-functors are dual Postnikov-functors.

REMARK. In the case of $\Phi=\pi$ the maps $k: X \rightarrow X_{\alpha}$ in (P1) can be realized by Postnikov-fibrations (i.e. by fibrations with an EilenbergMac Lane complex as fiber). In the dual case which we just treated the corresponding maps $k: X_{\alpha} \rightarrow X$ can be taken as Cartan-Serre-fibrations. For $\wp_{p_{E}}$, the category of css-spectra, the authors of [3] give a completely parallel treatment of Postnikov-decompositions and of Cartan-Serre-decompositions of a spectrum. There are many aspects in this duality which are not yet sufficiently explored.

8. Homology. We will consider the category $\mathfrak{F}_{*}$ of all simplyconnected $C W$-complexes and the corresponding homotopy-category $\mathfrak{F}_{s I I}$. We define the homology functor $H(\quad)=\left\{H_{m}(\quad)\right\}$ as well as $H^{(n)}=\left\{H_{m}\right\}_{m \leqq n}$ in the same way as the homotopy functor. We are now going to determine all weak dual Postnikov-functors for $H$ on $\mathfrak{F}_{s}$. Our first statement is again:

8.1. $\alpha=\left(H^{(n)}, T^{(n)}, P^{(n)}\right)$ is a dual weak Postnikov-functor to $H$ where $T^{(n)}$ and $P^{(n)}$ are the inclusions resp. the projection.

Obviously $\alpha$ is a $d$-functor. Take the category $\mathbb{R}_{\alpha}=\left\{X \mid H_{m}(X)=0\right.$, $m>n$. If $X \in \mathfrak{F}_{s}$ is an object, one has to construct a space $X^{\prime} \in \mathbb{R}_{k}$ and a map $k: X^{\prime} \rightarrow X$ such that $H_{m}(k)$ is an isomorphism for $m \leqq n$. This however is the purpose of a homology decomposition of a $C W$ complex (simply connected) and can be found in [2] This proves ( $\mathrm{P} 1)$.

In [2] there is also a counterexample which proves that $(\tilde{\mathrm{P}} 2)$ is not true in general for our triple $\alpha$. It is comparatively easy to verify on $\mathfrak{F}_{\text {s }}$ that all weak dual Postnikov-functors $\alpha=(\Psi, T, P)$ for

${ }^{1}$ In a standard notation this means $h=E^{n-2} \eta$, where $\eta: S^{3} \rightarrow S^{2}$ is the classical Hopf-map and $E$ is the suspension. 
$H$ are isomorphic to $\left(H^{(n)}, T^{(n)}, P^{(n)}\right)$ for suitable $n$ or to $(H, 1,1)$, 1: $H \rightarrow H$. We establish a proof of this in several steps:

8.2. If under our assumptions there is an $\bar{a} \in \Psi(X), \bar{a} \neq 0, T(\bar{a})=$ $a \in H_{n}(X)$, then $S^{n} \in \mathfrak{R}_{\alpha}$.

Proof. There is a space $X^{\prime} \in \mathfrak{P}_{\text {s }}$ and a map $f: X \rightarrow X^{\prime}$ with the following properties: $H_{m}(f)$ is a monomorphism for $m>n$ and $H_{m}\left(X^{\prime}\right)=0$ for $m<n$. One erects the cone over the $(n-1)$-skeleton of $X$ and for $f$ one takes the inclusion map. Since $H_{n}(f) a$ is in the range where the Hurewicz-theorem holds we get a map $f^{\prime}: S^{n} \rightarrow X^{\prime}$ such that $H\left(f^{\prime}\right) \beta=H(f) a$ for suitable $\beta \in H\left(S^{n}\right)$. We can assure the existence of a $\bar{\beta} \in \Psi\left(S^{n}\right)$ with $T(\bar{\beta})$. Thus $\beta=r \nu$, where $\nu \in H_{n}\left(S^{n}\right)$ is the fundamental class. Since there is a map $r: S^{n} \rightarrow S^{n}$ with $H(\bar{r}) \nu=r \nu=\beta$ and $H(\bar{r})$ is a monomorphism, we find a $\bar{\nu} \in \psi\left(S^{n}\right)$ with $T(\bar{\nu})=\nu$. This proves: $P: H\left(S^{n}\right) \rightarrow \psi\left(S^{n}\right)$ is an isomorphism.

8.3. Under the same assumptions as in 8.2. each $b \in H_{n}(Y)$ for arbitrary $Y \in \mathfrak{P}_{s}$ possesses a counterimage $\bar{b}$ under $T$, i.e., $T(\bar{b})=b$.

Proof. Carry out the same construction as in 8.2.: We obtain a $Y^{\prime}$ and $f: Y \rightarrow Y^{\prime}$ with the corresponding properties and accordingly a map $f^{\prime}: S^{n} \rightarrow Y^{\prime}$ such that $H\left(f^{\prime}\right) T(\bar{\nu})=H(f) b$. That proves that $H(f) b \in$ image $T$; and since $H_{n}(f)$ is a monomorphism, that $b \in$ image $T$.

8.4. Under the same assumptions as in 8.2. each $b \in H_{r}(Y), r \leqq n$ possesses a counterimage $\bar{b}$ under $T$.

Proof. Set $k=n-r, D^{n}=D=S^{r} \times S^{k}$. The fundamental classes in the homology groups of $S^{r}, S^{k}, D$ are resp. $s^{r}, s^{k}$, and $d^{n}$. It is clear that $d \in$ image $T$. We are going to prove that $s^{r}, s^{k} \in$ image $T$. There is by property ( $\left.\check{\mathrm{P}} 1\right)$ a map $h: R \rightarrow D$ where $R \in \mathfrak{Q}_{\alpha}$ and $\Psi(h)=$ isomorphism. There is furthermore a $b \in H(R)$ with $H(h) b=d$. Our statement would be proved as soon as we are certain that there are counterimages of $s^{r}$ and $s^{k}$ in $H(R)$ under $H(h)$ because $H(R) \approx \Psi(R)$ by assumption; and this is furnished by a simple cupproduct argument: Let $\bar{s}^{r}, \bar{s}^{k}$ and $\bar{d}$ be the corresponding cohomology elements to $s^{r}, s^{k}$, and $d$ in $H^{*}(D)$. One has $\bar{s}^{r} \cup \bar{s}^{k}=\bar{d}$. Assume $s^{r} \in$ image $H(h)$ ! This implies $H^{*}(h) \bar{s}^{r}=0$ which is impossible since

$$
H^{*}(h) \bar{d}=H^{*}(h) \bar{s}^{r} \cup H^{*}(h) \bar{s}^{k} \neq 0 .
$$

We are now in a position to prove the following theorem along 
the same line as we did for Theorems 3 and 4 .

Theorem 5. Every dual weak Postnikov-functor is isomorphic to $\left(H^{(n)}, T^{(n)}, P^{(n)}\right)$ or to $(H, 1,1), 1: H \rightarrow H$ on the category $\mathfrak{P}_{s}$.

9. Filtered spaces. Our next and last example comes again from topology. Take a category $\Re$ of based filtered spaces $X=$ $\left\{X_{n}\right\}, \cdots \subset X_{n} \subset X_{n+1} \subset \cdots X=\bigcup_{n \geqq 0} X_{n}$ and continous maps which preserve the filtration (i.e., $f\left(X_{n}\right) \subset Y_{n}$ for $f: X \rightarrow Y$ in $\Re$ ). We assume that with each collection $X_{\iota} \in \Re$ the wedge $V_{\imath} X_{\imath}$ is also contained in $\Re$. The category of $C W$-complexes may serve as an example. For future applications we assume the following condition:

(c) For $X, Y \in \Re$ and $x \in X_{n}, y \in Y_{n} x \notin X_{n-1}$ there is always a map $f: X \rightarrow Y$ (for $x \in X^{\prime} \subset X \in k$ ) in $\Re$ such that $f(x)=y$.

The background of condition (c) is that there are no other partitions in $\Omega$ of a fixed $X$ which are preserved by maps in the category. We will now deal with the functor $A: \Omega \rightarrow \Re$ which is defined by:

$$
\Lambda(X)=\bigvee X_{n} \quad X \in \Re
$$

for all $n$ such that $X_{n} \varsubsetneqq X$. If $f: X \rightarrow Y$ in $\Re$ one puts:

$$
\Lambda(f)=\mathrm{V} f_{n}, f_{n}: X_{n} \longrightarrow Y_{n}, f_{n}=f \mid X_{n} .
$$

We are going to construct all dual Postnikov-decompositions of $\Lambda$. To this end we introduce the functors $\Lambda^{(n)}: \Omega \rightarrow \Omega$ which are defind by $\Lambda^{(n)}(X)=\mathrm{V}_{m \leqq n} X_{m}$ and correspondingly for the maps. There is a transformation $T^{(n)}: \Lambda^{(n)} \rightarrow \Lambda$ (the inclusion $\mathbf{V}_{m \leqq n} X_{m} \subset \mathbf{V}_{m \geqq 0} X_{m}$ ) and a transformation $P^{(n)}: \Lambda \rightarrow \Lambda^{(n)}$ (the projection $\mathbf{V}_{m \geqq 0} X_{m} \rightarrow \mathrm{V}_{m \leqq n} X_{m}$ where all terms $X_{m}, m \geqq n+1$ are mapped into the base point) such that $P T=1$. This proves that $\Lambda^{(n)}$ is a dual $d$-functor to $\Lambda$. Accordingly $\mathfrak{Q}_{\alpha}$ (where $\alpha=\left(\Lambda^{(n)}, T^{(n)}, P^{(n)}\right)$ ) is the full subcategory of $\Re$ which consists of all spaces $X$ with $X_{n}=X$. We consider the inclusions $k: X_{n} \rightarrow X$ which induce by definition isomorphisms under $A^{(n)}$; this proves ( $\mathrm{P} 1)$. Now let $Y \in \mathfrak{R}_{\alpha}$ be any space and $k^{\prime}: Y \rightarrow X$ a map with $\Lambda^{(n)}\left(k^{\prime}\right)=$ isomorphism. In $r=k_{n}^{\prime}: Y_{n}=Y \rightarrow X_{n}$ we have a map with $k r=k^{\prime}$ which is moreover uniquely determined by that equation.

We have proved:

9.1. The triples $\alpha^{(n)}=\alpha=\left(\Lambda^{(n)}, T^{(n)}, P^{(n)}\right)$ are dual Postnikovfunctors for $\Lambda$,

Up to this point we have not used condition (c). Now let $\alpha=$ $(\Psi, T, P)$ be any Postnikov-functor $\Lambda$. Without affecting generality we may assume that $T: \Psi \rightarrow \Lambda$ is an inclusion i.e., $\Psi(X) \subset \Lambda(X)$, 
$\Psi(f)=\Lambda(f) \mid \Psi(X)$ for an $f \in \Omega$. By using condition (c) we find that there is not much choice for $\Psi(X)$ : It must be either $\Psi(X)=\Lambda(X)$ or $\Lambda^{(n)}$ for a certain $n$. We sum this up in the following theorem:

THEOREM 6. The triples $\alpha^{(n)}=\left(\Lambda^{(n)}, T^{(n)}, P^{(n)}\right)$ are dual Postnikovfunctors for $\Lambda$. They are the only dual Postnikov-functors for $\Lambda$ provided (c) holds.

ClOSING REMARKS. In our abstract theory of Postnikov-decompositions there are up to this point no $k$-invariants. The reason for this is that for such a system of invariants one needs a suitable cohomology. A construction of such a general cohomoly theory (on this abstract level) will be given in a later paper.

\section{REFERENCES}

1. F. W. Bauer and J. Dugundji, Categorical homotopy and fibrations, Trans. Amer. Math. Soc. (to appear).

2. E. H. Brown, Jr., and A. H. Copeland, Jr., An homology analogue of Postnikov systems, Michigan Math. J. 6 (1959), 313-330.

3. D. Burghelea and A. Deleanu, Resolution de Cartan-Serre et de Postnikov dans la categorie homotopique des spectres, C. R. Acad. Paris (A) 263 (1966), 361-364.

4. A. Dold, Halbexakte Homotopiefunktoren, Lecture Notes in Mathematics, 1965.

5. Donald W. Kahn, The spectral sequence of a Postnikov-system, Comment. Math. Helv. (3) $\mathbf{4 0}$ (1966).

6. J. C. Moore, Semi-simplicial complexes and Postnikov-systems, Sympos. intern. Mexiko, 1956.

7. M. M. Postnikov, Investigations in homotopy theory of continous mappings, Trudy

Stecklov 461955.

Received May 25, 1967.

Rice UnIVERSITy 


\section{PACIFIC JOURNAL OF MATHEMATICS}

\section{EDITORS}

\author{
H. ROYDEN \\ Stanford University \\ Stanford, California \\ R. R. Phelps \\ University of Washington \\ Seattle, Washington 98105
}

J. DUGUNDJI

Department of Mathematics

University of Southern California

Los Angeles, California 90007

RICHARD ARENS

University of California

Los Angeles, California 90024

\section{ASSOCIATE EDITORS}
E. F. BECKENBACH
B. H. NeumanN
F. WOLF
K. YosidA

\section{SUPPORTING INSTITUTIONS}

UNIVERSITY OF BRITISH COLUMBIA

CALIFORNIA INSTITUTE OF TECHNOLOGY

UNIVERSITY OF CALIFORNIA

MONTANA STATE UNIVERSITY

UNIVERSITY OF NEVADA

NEW MEXICO STATE UNIVERSITY

OREGON STATE UNIVERSITY

UNIVERSITY OF OREGON

OSAKA UNIVERSITY

UNIVERSITY OF SOUTHERN CALIFORNIA
STANFORD UNIVERSITY

UNIVERSITY OF TOKYO

UNIVERSITY OF UTAH

WASHINGTON STATE UNIVERSITY

UNIVERSITY OF WASHINGTON

AMERICAN MATHEMATICAL SOCIETY CHEVRON RESEARCH CORPORATION TRW SYSTEMS

NAVAL WEAPONS CENTER

Printed in Japan by International Academic Printing Co., Ltd., Tokyo, Japan 


\section{Pacific Journal of Mathematics}

\section{Vol. 26, No. $1 \quad$ November, 1968}

Efraim Pacillas Armendariz, Closure properties in radical theory......... 1

Friedrich-Wilhelm Bauer, Postnikov-decompositions of functors .......... 9

Thomas $\mathrm{Ru}-$ Wen Chow, The equivalence of group invariant positive definite

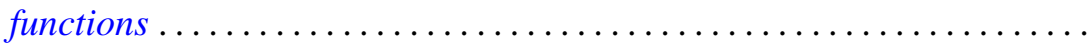

Thomas Allan Cootz, A maximum principle and geometric properties of

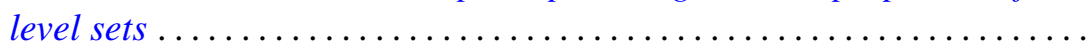

Rodolfo DeSapio, Almost diffeomorphisms of manifolds ............ 47

R. L. Duncan, Some continuity properties of the Schnirelmann density......

Ralph Jasper Faudree, Jr., Automorphism groups of finite subgroups of

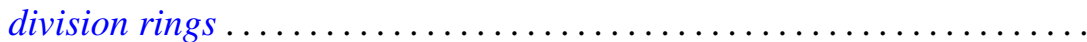

Thomas Alastair Gillespie, An invariant subspace theorem of $J$.

Feldman.........................................

George Isaac Glauberman and John Griggs Thompson, Weakly closed direct factors of Sylow subgroups .............................

Hiroshi Haruki, On inequalities generalizing a Pythagorean functional equation and Jensen's functional equation .....................

David Wilson Henderson, D-dimension. I. A new transfinite dimension.....

David Wilson Henderson, D-dimension. II. Separable spaces and

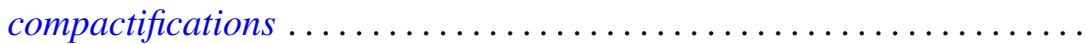

Julien O. Hennefeld, A note on the Arens products ............... 115

Richard Vincent Kadison, Strong continuity of operator functions ...

J. G. Kalbfleisch and Ralph Gordon Stanton, Maximal and minimal coverings of $(k-1)$-tuples by $k$-tuples.

Franklin Lowenthal, On generating subgroups of the Moebius group by pairs of infinitesimal transformations...

Michael Barry Marcus, Gaussian processes with stationary increments possessing discontinuous sample paths . .

Zalman Rubinstein, On a problem of Ilyeff ...

Bernard Russo, Unimodular contractions in Hilbert space. ...

David Lee Skoug, Generalized Ilstow and Feynman integrals...

William Charles Waterhouse, Dual groups of vector spaces . 